\title{
Mapping UML 2.0 Activities to Zero-Safe Nets
}

\author{
Sabine Boufenara ${ }^{1}$, Faiza Belala ${ }^{1}$, Kamel Barkaoui ${ }^{2}$ \\ ${ }^{1}$ LIRE Laboratory, Mentouri University of Constantine, Algeria; ${ }^{2}$ CEDRIC-CNAM, Rue Saint-Martin, Paris, France. \\ Email: sabineboufenara@yahoo.com,belalafaiza@hotmail.com,barkaoui@cnam.fr
}

Received February $18^{\text {th }}, 2010$; revised April 6 ${ }^{\text {th }}, 2010$; accepted April $6^{\text {th }}, 2010$.

\begin{abstract}
UML 2.0 activity diagrams (ADs) are largely used as a modeling language for flow-oriented behaviors in software and business processes. Unfortunately, their place/transition operational semantics is unable to capture and preserve semantics of the newly defined high-level activities constructs such as Interruptible Activity Region. Particularly, basic Petri nets do not preserve the non-locality semantics and reactivity concept of ADs. This is mainly due to the absence of global synchronization mechanisms in basic Petri nets. Zero-safe nets are a high-level variant of Petri nets that ensure transitions global coordination thanks to a new kind of places, called zero places. Indeed, zero-safe nets naturally address Interruptible Activity Region that needs a special semantics, forcing the control flow by external events and defining a certain priority level of executions. Therefore, zero-safe nets are adopted in this work as semantic framework for UML 2.0 activity diagrams.
\end{abstract}

Keywords: UML Activity Diagrams Formalization, Interruptible Activity Region, Zero-Safe Nets

\section{Introduction}

The Unified Modelling Language (UML) [1] has recently undergone a significant upgrade of its basic concepts, giving rise to a new major version, namely UML 2.0. Being widely used for specification and documentation purposes in the software development process, UML offers a spectrum of notations for capturing different aspects of software structure and behaviour. Activity diagram (AD) notations are intended to model behavioural aspects of software systems, particularly control and data flows.

Activity Diagrams (ADs) are widely used to model various types of applications fluctuating from basic computations to high level business processes, embedded systems and system-level behaviors. They facilitate the modelling of control and object (or data) flows by introducing a multitude of new concepts and notations such as collections, streams, loops and exceptions. Several semantics models have been defined to support these concepts. Nevertheless, many problems persist and reduce the usability of ADs [2,3]. This is mainly due to the new constructs and principles complexity and their formal semantics lack, leading to inconsistent interpretations of the model. For example, in a workflow process, described in terms of tasks and execution orders between them, Termination (or Cancelation) concept may be modeled via ADs Interruptible Activity Region. This modelling may have several interpretations since the used modelling concepts are still informal. Thus, a large gap has to be bridged prior to obtain an execution model and automated reasoning.

The abstract semantics of ADs have also completely changed in UML 2.0. They are no longer considered as a kind of state-machine diagrams and their semantics is being well explained in terms of Petri net concepts. But, basic Petri nets do not preserve semantics of new constructs of UML 2.0 ADs. We believe that this is essentially due to the locality character (local activations at transitions enabling) of basic Petri nets, whilst in UML 2.0, contrary to UML 1.x, the activation of computational steps may be not local.

Many attempts are currently led to give UML 2.0 ADs an operational semantics via some well known formal models such as high-level Petri nets [4], Abstract State Machine [5] and so on, for eventual analysis and simulation purposes. The objective of this paper is to describe how Zero Safe Nets (ZSNs for short) are very suitable to handle semantics of UML 2.0 ADs Interruptible Activity Region. ZSN is a new variant of Petri-net model introduced by Bruni [6] to define synchronization mechanisms among transitions without introducing any new interaction mechanism. On the basis of this formalism, we suggest a set of mapping rules to define a formal semantic of UML 2.0 ADs complex constructs. ZSNs semantics is then used to conduct control flow in the net guarantying atomicity and isolation of a transaction that is all what we need in the cancellation schema. This formal specification 
is precise enough to enable a unique model interpretation at an utmost detail level. It can therefore serve as tools implementation basis. Finally, it participates to ensure that the specified behaviour meets the intended intuition of the modeller.

The remainder of this paper is structured as follows. A detailed discussion of related works is given in Section 2 . Section 3 presents syntax and informal semantics of both Interruptible Activity Region in UML 2.0 ADs and ZSNs. In Section 4, we describe our contribution by presenting first the problematic, then the intuitive mapping to address the Interruptible Activity Region formalization and finally the formal definition of this mapping. Section 5 concludes the paper with remarks and outlook on future work.

\section{Related Works and Paper Contribution}

The state of the art concerning UML 2.0 activity diagrams semantics covers three different approaches: the first based on Petri-nets, the second using graph transformation rules and the third generating pseudo-code. Since the two latter approaches are out of the scope of the present paper, we therefore discuss UML 2.0 semantics related work only in terms of Petri-nets.

Since UML specification envisions a "Petri-like semantics" for activity diagrams, it is quite interesting to propose a mapping between the two notations. Barros [7] suggests translating a subset of ADs concepts to Petri nets ones. Actions considered as activities in Petri nets are no longer atomic, inducing to ADs semantics violation in the UML specification standard ([1] p. 203). Moreover, only locally behaved activities are considered in Petri nets, whereas non-locality semantics is one major innovative characteristic of UML 2.0 ADs.

In [8], an $\mathrm{AD}$ is transformed into FMC (Fundamental Modeling Concepts) for their attractive feature, and then, a Colored Petri net is constructed for execution and validation purposes. This approach focuses on abstract syntax and thus, does not preserve semantics, especially for the atomicity principle.

Störrle uses different variants of Petri nets (from colored to procedural and exception Petri nets) to propose a formal semantics to UML 2.0 ADs. The author tackles the formalization of many concepts [9-12] such as controlflow, procedure calling, data-flow, exceptions, loopnodes, conditional-nodes and expansion-regions using various versions of Petri nets. However, different concepts can generally coexist in the same AD. Therefore, analysis of the whole system behavior is not possible due to non-unified formalism.

The development culminates in [13-15] concluding that Petri-nets might, after all, not be appropriate for formalizing activity diagrams. Especially, mapping advanced concepts, such as interruptible activity regions, is found to be not intuitive. Moreover, Petri-nets formalization of
ADs concepts is not unified and integrates different variants of Petri-nets to map concepts belonging to the same diagram. Additionally the traverse-to completion semantics insurance is identified as being the major problem in Petri-nets mapping. In [16], we have proposed a generic mapping from UML 2.0 ADs to Zero-Safe Nets (ZSNs) and have shown by several examples how this Petri net variant can surmount this latter problem. Indeed, non-locality semantics of ADs is preserved via a global synchronization, offered by ZSNs, rather than a local one as in basic Petri nets. In [17], we have been interested by streaming parameters and exception outputs constructs in UML 2.0 ADs, their formal semantics has been defined in terms of these Petri nets variant without losing an important characteristic of those concepts that is atomicity.

In this paper, we extend these recent works by improving the proposed mapping to be more formal and general. Indeed, we examine semantics of Interruptible Activity Region construct in which actions need to be promptly cancelled on the reception of an external event. This can not be provided with basic Petri nets that are local and not reactive.

\section{Basic Concepts}

We are interested in this section to remind fundamental notions used in this study. For more details, the reader can consult $[1,18]$ (for Interruptible Activity Region in ADs) and [6] (for ZSNs).

\subsection{Interruptible Activity Region}

The UML 2.0 specification made by the OMG [1] standard provides a meta-model to define the abstract syntax for activity diagrams including Interruptible Activity Region.

These ADs special regions are groups of nodes where all execution might be terminated, if an edge traverses an interruptible activity, before leaving the region. Interrupting edges must have their source node in the region and their target node outside it, but in the same AD containing the region.

An Interruptible Activity Region is notated by a dashed, round-cornered rectangle drawn around the nodes contained in the region. An interrupting edge is notated with a lightning-bolt activity edge.

During the process of an Interruptible Activity Region, the reception of an event (exception-event) triggers the block abort of that part of the Activity, and resumes execution with another action that may be the exception-handler. The standard specification [1] states, that "When a token leaves an interruptible region via edges designated by the region as interrupting edges, all tokens and behaviours in the region are terminated". Interruptible regions are introduced to support more flexible non-local termination of flow. 
Example 1. Figure 1 gives an example taken from [1] illustrating these concepts. This example illustrates that if an order cancellation request 'Order Cancel Request' is made, while executing one of the actions (receive, fill, or ship orders), the 'Cancel Order' behaviour is immediately invoked and the action being executed is aborted.

Cancellation is a very common behavior in the execution of workflows process. It is used to capture the interference of an event or an activity in other activities execution of a workflow preventing execution or termination. A cancellation can involve a cancellation area, a sub process or an entire workflow. In UML 2.0 ADs, Interruptible Activity Region has been defined to hold such behavior.

\subsection{Introducing ZSNs}

Zero-safe nets have been introduced by Bruni in [6] to define synchronization mechanism among transitions, without introducing any new interaction mechanism besides the ordinary token-pushing rules of nets. Their role is to ensure the atomic execution of complex transitions collections, which can be considered as synchronized.

Atomic execution of multiple coordinated transitions is forth possible in ZSNs thanks to a new kind of places, called zero places. From an abstract viewpoint, those transitions will appear as synchronized. Zero places are bound to zero tokens in a system observable state. A token in a zero place is equivalent to a system internal state that is non-observable. ZSN synchronized evolution must begin at an observable state, evolve in non-observable markings and must end at an observable state. Therefore, ZSNs define two sorts of places; stable places corresponding to net places, and zero places. A ZSN evolution is considered as a transaction. A stable token generated in a transaction is frozen all over the evolution; it is released only once the transaction is finished. We notice that a transaction in this case, is represented by a system activity possibly composed of a set of concurrent but atomic sub-activities.

Zero places coordinate the atomic execution of transi-

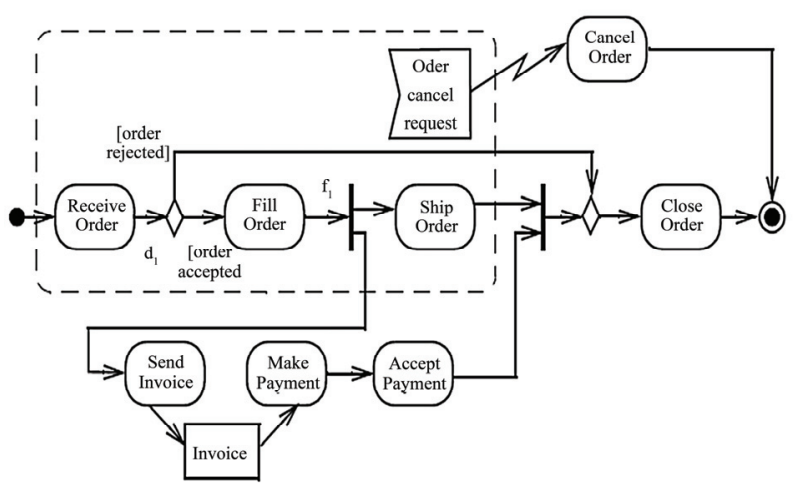

Figure 1. Interruptible Activity Region enclosed by a dashed line. The Interrupting edge is expressed by a lightning-bolt style tions which, from an abstract viewpoint, will appear as synchronized. At the abstract level, we are not interested in observing the hidden state. Modeling of the well- known example of 'dining philosophers' problem' is sufficient to show how ZSNs are powerful to synchronize transitions in an atomic way (see [19] for more details).

Example 2. (taken from [19]). There are $n$ philosophers (here, we suppose $n=2$ ) sitting on a round table; each having a plate in front and between each couple of plates there is a fork, with a total of $n$ forks on the table. Each philosopher cyclically thinks and eats, but to eat he needs both the left hand side fork and the right hand one of his plate. After eating a few mouthfuls, the philosopher puts the forks back on the table and starts thinking again.

It is not difficult to imagine conflict situations leading to a deadlock when each philosopher takes one fork and cannot continue. This is due to the fact that the coordination mechanism is hidden inside transitions $\left(\right.$ Take $_{1}$ and $\mathrm{Take}_{2}$ ) that are too abstract (see Figure 2(a) modeling a centralized non-terminism). Places $\mathrm{Fk}_{\mathrm{i}}$ denote forks. A token in the place $\mathrm{Fk}_{\mathrm{i}}$ means that the ith fork is on the table.

The same model is redrawn using free choice nets. Decisions are now local to each place i.e. decisions are made independently (see Figure 2(b)) and deadlock situation is clear. One decision concerns the assignment of the first fork whether to the first or to the second philosopher, the other decision concerns the assignment of the second fork. Note that $\mathrm{Ch}_{\mathrm{i}, \mathrm{j}}$ stands for Choicei, $j$ where $i{\text { denotes } \text { Fork }_{i} \text { and } j \text { denotes PHilosopher }}_{j}$. Then, it might happen that the first fork is assigned to the first philosopher $\left(\mathrm{Ch}_{1,1}\right)$ and the second fork is assigned to the second philosopher $\left(\mathrm{Ch}_{2,2}\right)$, and in such case the free choice net deadlocks and none of the Take $e_{i}$ actions can occur. Thus, the translated net admits non-allowed computations in the abstract sub-system of Figure 2(a).

Zero-safe nets surmount this deadlock problem by executing only some atomic transactions, where tokens produced in low-level resources are also consumed. In the example, the ow-level. This is possible, but at the expense of preseinvisible resources consist of places $\mathrm{Fk}_{\mathrm{i}, \mathrm{j}}$ for $1 \leq \mathrm{i}, \mathrm{j} \leq 2$, that can be interpreted as zero places. In this way the computation performing $\mathrm{Ch}_{1,1}$ and $\mathrm{Ch}_{2,2}$ is forbidden, because it stops in an invisible state, i.e., a state that contains zero tokens (see Figure 2(c)).

While basic Petri nets fail to conserve the system semantics at a low-level, free choice nets make local decisions possible at lrving execution semantics. Zero-safe nets are able to preserve execution semantics even when expressed in refined way.

\section{Formal Definitions [6]}

A ZSN is a 6-tuple $B=\left(S_{B}, T_{B}, F_{B}, W_{B}, u_{B}, Z_{B}\right)$ where $N_{B}$ $=\left(\mathrm{S}_{\mathrm{B}}, \mathrm{T}_{\mathrm{B}}, \mathrm{F}_{\mathrm{B}}, \mathrm{W}_{\mathrm{B}}, \mathrm{u}_{\mathrm{B}}\right)$ is the underlying place/ transition 


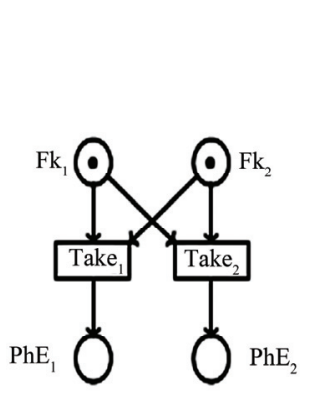

(a)

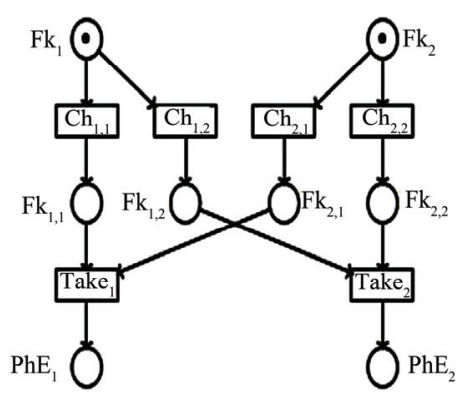

(b)

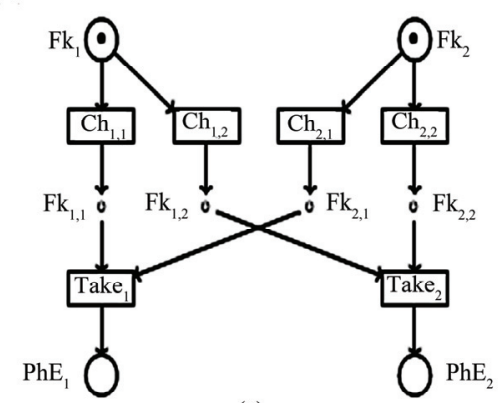

(c)

Figure 2. Example of dining philosophers: (a) Centralized nondeterminisme, (b) Local nondeterminisme presenting deadlock, (c) Atomic free choice

net. $\mathrm{S}_{B}$ is a non-empty set of places. $T_{B}$ is a non-empty set of transitions. $F_{B} \subseteq\left(S_{B} \times T_{B}\right) \cup\left(T_{B} \times S_{B}\right)$ is a set of directed arcs. $\mathrm{W}_{\mathrm{B}}$ is the weight function that associates a positive integer to each arc. $u_{B}$ is the places marking associating positive tokens number to each place. $\mathrm{Z}_{\mathrm{B}} \subseteq \mathrm{S}_{\mathrm{B}}$ is the set of zero places (also called synchronization places). The places in $S_{B} \backslash Z_{B}$ are stable places. A stable marking is a multiset of stable places. The presence of one or more zero places of a given marking makes it unobservable, while stable markings describe observable states of the system.

Let $B$ be a zero safe net and let $s=\mathrm{u}_{0}\left[\mathrm{t}_{1}>\mathrm{u}_{1} \ldots \mathrm{u}_{\mathrm{n}-1}\left[\mathrm{t}_{\mathrm{n}}>\mathrm{u}_{\mathrm{n}}\right.\right.$ be a firing sequence of the underlying net $\mathrm{N}_{B}$ of $\mathrm{B}$,

- The sequence $s$ is a stable step of B if:

$$
\begin{aligned}
& \forall a \in S_{B} \mid Z_{B}, \Sigma^{n}{ }_{i=1} \operatorname{pre}\left(t_{i}\right)(a) \leq u_{0}(a) \\
&(\text { Concurrent enabling) }
\end{aligned}
$$$$
\text { (Stable fairness) }
$$

$\operatorname{Pre}(\mathrm{t})(a)$ defines the weight of the arc from place $a$ input of transition $t$ to this one. $\operatorname{Post}(\mathrm{t})(a)$ defines the weight of the arc from transition $t$ to its output place $a$. The concurrent enabling property insures the initial simultaneous enabling of all step transitions by stable places and not only those transitions allowing the initial triggering of the first execution. We notice that this property prohibits the consummation of stable tokens produced in the step by its transitions.

- Stable step $s$ is a stable transaction of B if in addition:

$$
\begin{aligned}
& \text { Markings } u_{1}, \ldots, u_{n-1} \text { are not stable } \\
& a \in S_{B} \mid Z_{B}, \sum^{n}{ }_{i=1} \operatorname{pre}\left(t_{i}\right)(a)=u_{0}(a)
\end{aligned}
$$

(Perfect enabling)

The perfect enabling ensures the consummation of all initial stable tokens before the transaction ends.

In a stable transaction, each transition represents a micro-step carrying out the atomic evolution through invisible states. Stable tokens produced during the transaction become active in the system, only at the end of the transaction.

Example 3. Consider the zero-safe net example of Figure 2(c). The firing sequence $\left\{\mathrm{Fk}_{1}, \mathrm{Fk}_{2}\right\}\left(\mathrm{Ch}_{1,1}>\left\{\mathrm{Fk}_{1,1}\right.\right.$, $\left.\mathrm{Fk}_{2}\right\} \quad\left(\mathrm{Ch}_{1,1}>\left\{\mathrm{Fk}_{2,2}, \mathrm{Fk}_{1,1}\right\}\right.$ is not a stable step since the stable fairness is not satisfied. The marking $\left\{\mathrm{Fk}_{2,2}, \mathrm{Fk}_{1,1}\right\}$ enables no transition, defining hence a deadlock situation. Since the sequence above is not a stable step and deadlocks at a non-visible state, so it is forbidden.

The two following firing sequences are the unique stable transactions:

$$
\begin{aligned}
& \left\{\mathrm{Fk}_{1}, \mathrm{Fk}_{2}\right\}\left(\mathrm{Ch}_{1,1}>\left\{\mathrm{Fk}_{1,1}, \mathrm{Fk}_{2}\right\}\left(\mathrm{Ch}_{2,1}>\left\{\mathrm{Fk}_{2,1}, \mathrm{Fk}_{1,1}\right\}\left(\mathrm{Take}_{1}\right.\right.\right. \\
> & \left\{\mathrm{PhE}_{1}\right\} . \\
& \left\{\mathrm{Fk}_{1}, \mathrm{Fk}_{2}\right\}\left(\mathrm{Ch}_{1,2}>\left\{\mathrm{Fk}_{1,2}, \mathrm{Fk}_{2}\right\}\left(\mathrm{Ch}_{2,2}>\left\{\mathrm{Fk}_{1,2}, \mathrm{Fk}_{2,2}\right\}\left(\mathrm{Take}_{2}\right.\right.\right. \\
> & \left\{\mathrm{PhE}_{2}\right\} .
\end{aligned}
$$

In what follows, we exploit features offered by zero-safe nets to define a priority level in ADs actions executions, leading to the reactivity definition.

\section{Handling Interruptible Activity Region via ZSNs}

Formalizing ADs using Petri nets seems to be a good approach. The specification states that "Activities are redesigned to use a Petri-like semantics" [1]. Unfortunately, basic Petri nets present some limits.

In [16], we have shown that Petri nets, supposed to be a semantic framework for ADs, are not well suitable to handle new UML semantics such as traverse-to- completion principle. Indeed, the latter requires a global synchronisation and not a local one as defined by Petri nets. We defined a generic mapping from ADs to zero-safe nets that preserves ADs operational semantics while focusing on traverse-to-completion principle and synchronization of fork and join nodes. Therefore, we covered control/data flows and concurrency. Besides, in [17], we have focused on semantics of streaming parameters and exception outputs, and showed also that ZSNs are able to express such complex semantics. Atomic transactions have been defined in ZSNs under a token game based on freezing tokens that have been created in the transaction until it ends. This becomes possible thanks to the zero-places.

The contribution of this paper is to define a suitable mapping of ADs to ZSNs, dealing with more complex concepts of UML 2.0 ADs, namely the Interruptible Activity Region. We show how basic Petri nets are not able 
to express semantics of this construct due to their non reactive aspect. We define a new net called $Z S N_{I A R}$ based on ZSNs, formalizing the Interruptible Activity Region as well as other important ADs principles and constructs such as global synchronisation of concurrent regions [16] and streaming parameters and exception outputs [17].

\subsection{Petri Nets Limits}

When dealing with the Interruptible Activity Region, two questions are to be considered: the first is about the raising and handling of exceptions and the second concerns the reactivity to external event.

1) Exceptions are a key example of non-local behavior. Raising and handling an exception means switching, from one of specified program states, to some other ones in one step (a kind of multi-goto)

In Petri nets, while system state is modeled via distributed marking over the whole net places, state changes are local. When mapping Interruptible Activity Region into Petri nets, state is hence distributed over many places of the region. To handle the cancellation semantics via Petri nets, we need to remove a set of place markings (of the interruptible region) at once. Moreover, the number of destructed tokens is only known at run time.

Yet, we can create some net structure warranting that all possible token distributions over places are covered. This is possible by adding arcs that will be connected to all potential combinations of all places in the region. It is obvious that this chaotic solution leads to a huge arcs number (spaghetti arcs). This, will greatly reduce the readability and understanding of such net. Reset arcs seem to be a good solution.

2) The reception of an external event triggers the activity block abortion in Interruptible Activity Region, and continues execution with another action that may be the exception-handler.

All actions of the Interruptible Activity Region are immediately aborted and no action outside the interruptible region can be executed before the handling of that event. This leads to a priority and isolation of execution.

Within the Petri nets semantics, there is no priority in executing two concurrent transitions. The choice of firing one of the enabled concurrent transitions is non-deterministic.

Example 4. In Figure 3, we give a naïve basic Petri net that formalizes the AD of Figure 1. The transcription follows mapping rules defined by Storrle in [3] (See Table 1). The author added a number of transitions, modeling the interruption event, equal to the cancelled actions in the region. Each transition is connected to the input place of a cancelled action and to transition Cancel Order via an output common place. When the Cancel Order Request is made, places of the Interruptible Activity Region, with dark gray, have to be emptied.
Table 1. Mapping rules from UML activities to basic Petri nets [3]
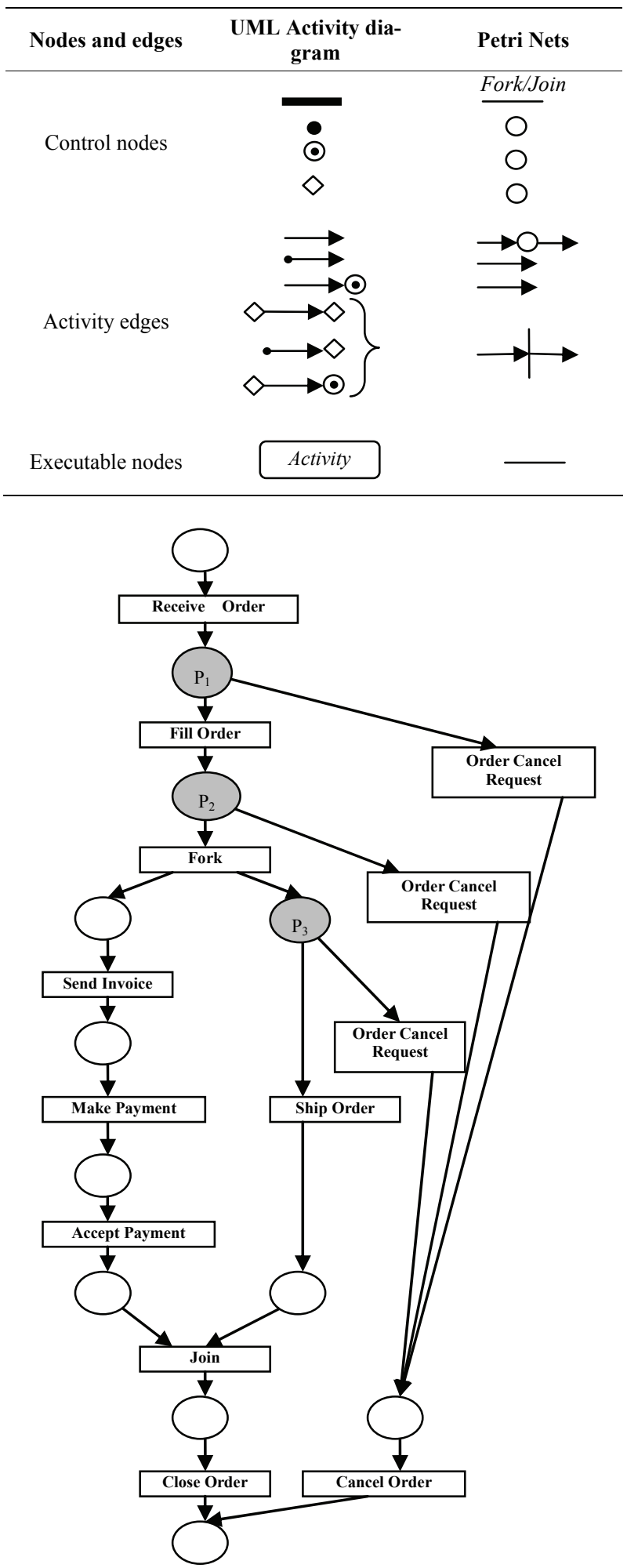

Figure 3. Intuitive mapping of the AD of figure 1 to basic Petri nets [3] 
We notice that semantics of cancellation (first point) does not appear in the net: First, the cancel event is not visible (sketched by transitions Order Cancel Request that are enabled by internal places) and second, the net is supposed to be 1-safe.

Regarding the second point mentioned above: when place $p_{I}$ is marked, both transitions Fill Order and Order Cancel Request are enabled and have the same probability to fire (situation of effective conflict). Whereas in this context, we would like to fire the aborting transition, that is Order Cancel Request.

The ZSNs model offers transitions coordination thanks to zero places. It guarantees atomicity and isolation of transaction, and this is all what we need in the cancellation schema. In what follows, we use ZSNs semantics to conduct the control flow in the net.

\subsection{Mapping Intuition}

In what follows, we discuss two zero-safe nets based approaches to formalize $\mathrm{AD}$ interruptible region with regard to semantics via the running example 1 .

The first solution introduces reset arcs and no new mechanism is necessary beyond the zero-safe nets semantics. In the net of Figure 4, we introduce a transition called 'cancel', and then we connect all places in the Interruptible Activity Region to that transition by a reset arc for each. The firing of transition 'cancel' empties all its input places at once, regardless of their marking. Thus, the net is no longer forced to be 1-safe. To overcome the second shortcoming pointed out, we add an input place 'interface place' to transition 'cancel'. This place represents the external cancel event. It is connected to transition 'cancel' via an arc of weight 1 . When the place 'interface place' is marked, the transition 'cancel' is enabled. Possibly, other transitions of the region are enabled at the same time. We need to guide the control to fire transition 'cancel' first. This is known as isolation and atomicity. To achieve this, we assume that 'interface place' is a zero place and not a stable one, so when marked, transition 'cancel' is enabled and immediately fired. This is due to the enabling property of ZSNs. Then another problem arises: when combining both solutions i.e. reset arcs and the interface zero place, enabling of transition cancel is made via the zero place connected with a non-reset arc. Thus, if another input place that has to be emptied by cancel, has an other output transition, it could be possible to fire that transition first and then 'cancel' transition indeterminably without impeding ZSNs rules. This is essentially caused by the presence of reset arcs. To overcome this problem, we can easily create a stable token in the transaction that is frozen until the transaction ends. The corresponding place is also an input one to cancelled transitions via reset arcs. (see Figure4).

In Figure 4, firing the external transition creates one stable token in the stable place $p_{\text {freeze }}$ and one zero token in

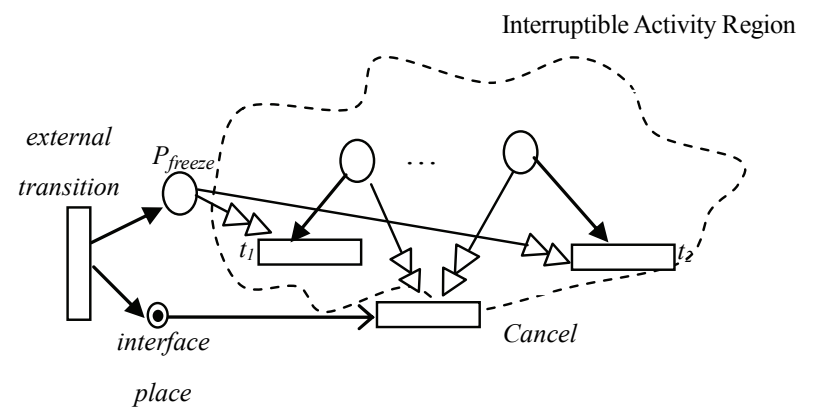

Figure 4. Formal semantics of the Interruptible Activity Region via ZSNs augmented with reset arcs

interface place. The stable token cannot be consumed until the transaction ends, hence prohibiting the firing of the region enabled transitions such as $t_{l}$ and $t_{2}$. The unique transition that satisfies firing conditions is cancel. The created token in $p_{\text {freeze }}$ can be consumed in the first next firing not being a cancellation procedure.

It is clear that such construction greatly improves modeling cancellation patterns and preserves semantics. However, adopting such technique has its drawbacks; the number of used reset arcs in this model depends always on the number of places in the interruptible region. This reduces considerably the net readability.

In Figure 5, we define a special cancellation transition cancel (pictured by an underlined rectangle) with its new enabling and firing semantics. Cancel may have many stable inputs and one zero input place, that is interface place. There are two different conditions to enable transition cancel:

1) Necessary condition but not sufficient to fire cancel: the input zero place is marked.

2) Effective firing condition: the instantaneous marking of cancel input places, i.e., input places markings when the zero token is created. This marking is calculated at run time, and this one is the enabling marking. Thus, once firing cancel transition, all of its input places are emptied.

When the zero place is marked (via an external transition), cancel is enabled, the current marking is then calculated

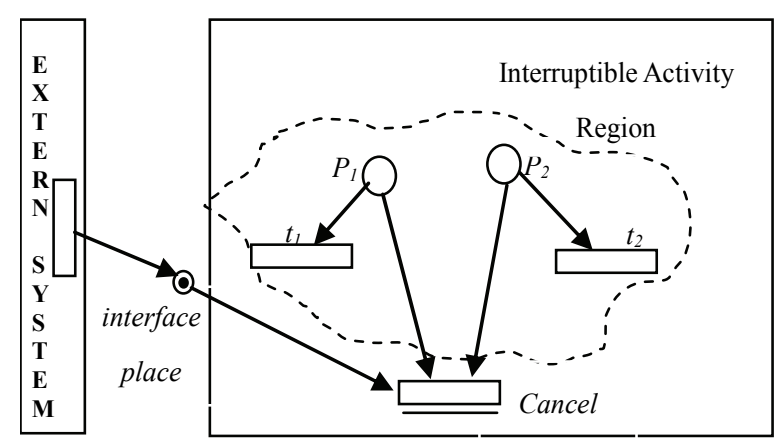

Figure 5. Formal semantics of the Interruptible Activity Region via ZSNs and a special transition cancel 
and is equal to the destroyed tokens. This latter is necessary to the transition firing. Hence, it is forbidden to fire $t_{l}$ or $t_{2}$ first. In such case, the cancel firing condition would not be satisfied leading to a deadlock situation in an invisible state (non-observable marking). Firing cancel will switch, from one of specified program states $\left(\left\{p_{1}\right\},\left\{p_{2}\right\}\right.$ or $\left.\left\{p_{1}, p_{2}\right\}\right)$, to some other ones in one step.

In our proposed semantics, event triggering cancellation is not formalized via a transition (this should be the intuition), but via a zero place. Hence, coordinating the execution of the termination action is made possible.

With basic Petri nets, this is not possible since it is agreed that an enabled transition can be fired or not, i.e. firing one of two concurrently enabled transitions is nondeterminitic. With ZSNs, interface place is modeled with a zero place rather than a stable one. Whenever, an out-transition (a transition not belonging to the system) is fired, a zero token is created in the interface place indicating that the system is actually executing a transaction. Transactions have a higher execution priority compared to transitions. Hence, firing cancel transition is prior to any other transition.

Figure 6 presents the mapping of the Interruptible Activity Region part of Figure 1. When Ship Order is enabled, a cancellation event occurs. This is traduced by marking the zero place interface place. The effective firing condition of cancel is calculated and it is equal to interface place, $\left.p_{3}\right\}$. Two transitions are now enabled: Ship Order and Cancel. Firing transition Cancel is prior than transition Ship Order. Firing Ship Order first, leads to a deadlock

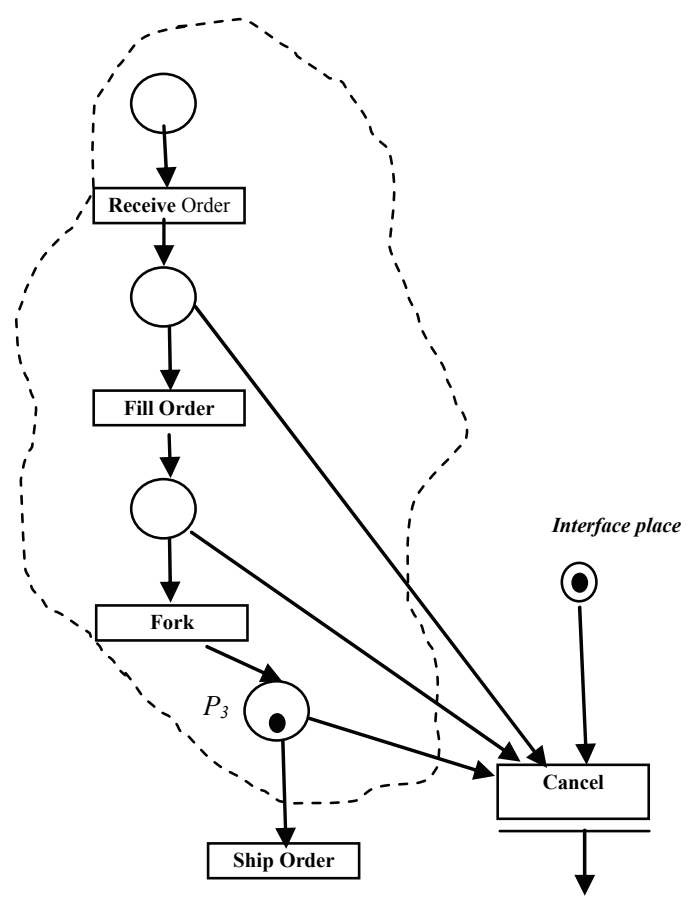

Figure 6. Intuitive mapping of the Interruptible Activity Region of the AD of Figure 1 to ZSNs situation (non finishing transaction) caused by the consumption of cancel transition enabling tokens.

\subsection{Formal Mapping}

Table 2 defines preliminarily hints on formalizing UML 2.0 ADs via ZSNs. This generic mapping covers basic constructs, concurrent-region, traverse-to-completion principle, streaming parameters, exception outputs and the Interruptible Activity Region.

Executable and fork/join nodes are mapped to transitions. Control nodes become stable or zero places, depending of the synchronization schema to be modeled. Specific Petri nets models are given in particular cases such as streaming parameters, exception outputs and the Interruptible Activity Region. Most of these notations have already been examined in earlier work. The semantics of the Interruptible Activity Region is discussed in this paper.

To formalize the mapping, we propose, for both basic activity diagram AD of UML 1.x and a complete one of UML 2.0, rigorous notations as given below. Extended activity diagram AD2 encloses new constructs and semantics, namely object nodes, traverse-to-completion principle, streaming parameters, exception outputs and Inturruptible Activity Region. Next, we define a formal semantic definition of AD2 in terms of ZSNs.

\section{Definition 1:}

An activity diagram is defined by a tuple $A D=(E N, B N$, $C N$, $i N, f N, C F$ ) where:

$E N$ : denote Executable Nodes, i.e., elementary actions. $E N=\left\{\mathrm{A}_{1}, \mathrm{~A}_{2}, \ldots, \mathrm{A}_{\mathrm{n}}\right\}$.

$B N$ : denote Branch Nodes i.e. decisions and merges. $B N=\left\{\mathrm{d}_{1}, \ldots, \mathrm{d}_{\mathrm{k}} ; \mathrm{m}_{1}, \ldots, \mathrm{m}_{\mathrm{k}^{\prime}}\right\}$, such as $: \mathrm{k} \geq \mathrm{k}^{\prime}$.

$C N$ : denote Concurrency Nodes i.e. forks and joins. $C N=\left\{\mathrm{f}_{1}, \ldots, \mathrm{f}_{\mathrm{m}} ; \mathrm{j}_{1}, \ldots, \mathrm{j}_{\mathrm{m}^{\prime}}\right\}, \quad$ such as: $\mathrm{m} \geq \mathrm{m}^{\prime}$.

$i N$ : denotes the initial Node.

$f N$ : denotes the final Node.

$C F$ : is a function denoting Control Flows. $C F \subseteq((E N$, $B N, C N, i N) \times(E N, B N, C N, f N))$. A directed arc sketches the control flow where the source may be an action, a branch, a control or the initial node and the arc target may be an action, a branch, a control or the final node.

Definition 2:

An UML2.0 AD is defined by a tuple $A D 2=(A D, O N$, $O F, C R, S A, E A, I A R)$ where:

$A D$ : is the corresponding basic activity diagram as defined above.

$O N$ : denotes Object Nodes. In this work, we deal with pins. $O N=\left\{\mathrm{o}_{1}, \ldots, \mathrm{o}_{\mathrm{r}}\right\}$. Objects may represent data or streams $\left\{\mathrm{s}_{1}, \ldots, \mathrm{s}_{\mathrm{r}^{\prime}}\right\}$ or exceptions $\left\{\mathrm{e}_{1}, \ldots, \mathrm{e}_{\mathrm{w}^{\prime}}\right\}$.

$O F$ : is a function denoting Object (token) Flows. $O F$ $\subseteq((B N, C N, i N, O N) \times(B N, C N, f N, O N)) . O F=$ $\left\{o f_{1}, \ldots o f_{x}\right\}$. As tokens move across an object flow edge, they may undergo transformations. An object flow might carry a transformation behavior denoted $t b$. 
Table 2. The intuition of ADs formal semantics via ZSNs: Zero places are pictured with small circles

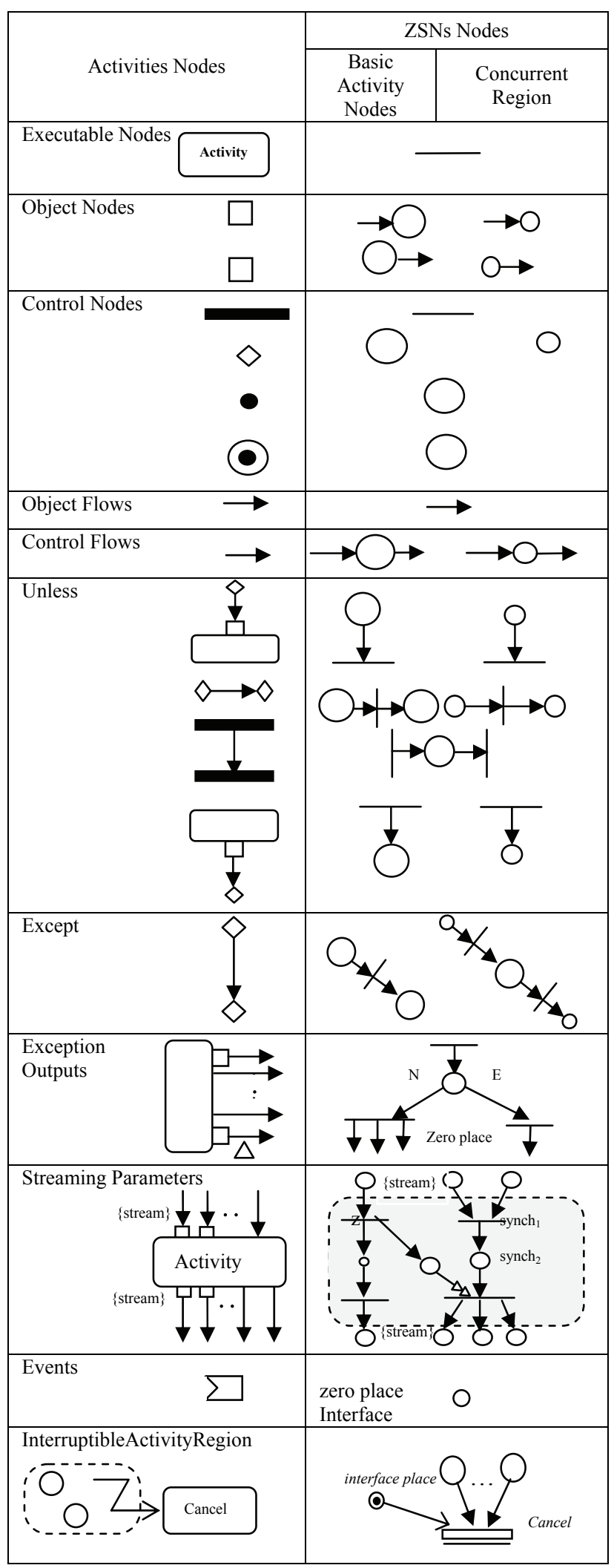

$C R$ : denotes Concurrent Regions. A concurrent region is a $s u b-A D 2$ delimited with a fork and a join. Concurrent regions have a special semantics under the traverse-tocompletion principle. This one has been discussed in [16] along with a generic mapping to ZSNs.

$S A$ : is a set of Streaming Actions $\left\{\mathrm{S}_{1}, \ldots, \mathrm{S}_{\mathrm{r}}\right\}$. A streaming action is an elementary action $A_{\mathrm{i}}$ with input/output streaming parameters $\mathrm{s}_{\mathrm{i}}$.

$E A$ : is a set of Exception Actions $\left\{\mathrm{E}_{1}, \ldots, \mathrm{E}_{\mathrm{w}}\right\}$. An exception action is an elementary action $A_{\mathrm{i}}$ having exception outputs $\mathrm{e}_{\mathrm{i}}$.

IAR: denotes Interruptible Activity Regions. An interruptible region is a $s u b-A D 2$ bound to a special specification Spec that can, informally, be Spec $(I A R)=($ evt, can$c e l)$ where $e v t$ is the interruption triggering event and cancel is the cancellation action. We note that evt and cancel do not belong to the $I A R$. In the perspective of cancellation, only actions $E N$ and control nodes $C N$ may be interrupted, thus the enclosed actions and control nodes define an IAR. $\mathrm{IAR}_{\mathrm{i}}=\left\{\mathrm{A}_{\mathrm{g}}, \ldots, \mathrm{A}_{\mathrm{g}}, \mathrm{f}_{\mathrm{h}}, \ldots, \mathrm{f}_{\mathrm{h}} ; \mathrm{j}_{\mathrm{v}}, \ldots\right.$, $\left.\mathrm{j}_{\mathrm{v}^{\prime}}\right\}$, such that, $\mathrm{A}, \mathrm{f}$ and $\mathrm{j}$ stand respectively for actions, forks and joins enclosed in the region.

For the sake of the presentation, we restrict our ZSN definition purpose to control flow, data flow and Interruptible Activity Region. Let $A D_{I A R}$ be a $s u b-A D 2$, such that, $\left.A D_{I A R}=(E N, B N, C N, i N, f N, C F, O N, O F)\right)$ and let Spec be a specification such that:

Spec $(I A R)=($ evt , cancel $)$. We define $A D_{I A R}$ by identifying, in addition to IAR nodes, the input and output object and branch nodes connected to each IAR node via a control or object edge, including edges. Recall that an $I_{A R}=\left\{A_{g}, \ldots, A_{g^{\prime}}, f_{h}, \ldots, f_{h^{\prime}} ; j_{v}, \ldots, j_{v^{\prime}}\right\}$.

Next, we define a formal mapping from a sub $A D 2$ $A D_{\text {IAR }}$ to a zero-safe net $Z S N_{I A R}$.

Example 5. Consider the AD of Figure 1: let $\mathrm{IAR}_{1}$ be $\mathrm{IAR}_{1}=\left\{\right.$ ReceiveOrder, FillOrder, ShipOrder, $\left.\mathrm{f}_{1}\right\}$, where $\mathrm{f}_{1}$ stands for the fork node and ReceiveOrder, FillOrder, ShipOrder $\} \subset$ EN.

We define $A D_{\text {IAR } 1}$ by identifying inputs and outputs of $\mathrm{IAR}_{1}$ nodes. $\mathrm{AD}_{\mathrm{IAR} 1}=\{$ ReceiveOrder, FillOrder, ShipOrder, $\mathrm{f}_{1}, \mathrm{~d}_{1},\left\langle\right.$ ReceiveOrder, $\left.\mathrm{d}_{1}\right\rangle,\left\langle\mathrm{d}_{1}\right.$, FillOrder $\rangle$, $\langle$ FillOrder, $\left.\mathrm{f}_{1}\right),\left\langle\mathrm{f}_{1}\right.$, ShipOrder $\left.\rangle\right\}$ where $\mathrm{d}_{1}$ stands for the decision node and pairs of the form $\langle\mathrm{x}, \mathrm{y}\rangle$ stand for edges such as $\mathrm{x}$ is the edge source and $y$ is its target. Spec $\left(\mathrm{IAR}_{1}\right)=(\mathrm{Or}-$ derCancelRequest, CancelOrder) where OrderCancelRequest stands for evt and CancelOrder for Cancel.

\section{Definition 3:}

$Z S N_{I A R}$ is a special ZSN defining the semantic of $A D_{I A R}$, an UML 2.0 sub activity diagram with the Spec specification.

$Z S N_{I A R}=\left(Z S N, S_{I A R}, Z_{\text {cancel }}\right.$, Cancel, ip, sp) where:

- There is a single source place ip, such that, ip $\in S_{B},{ }^{\bullet} i p=\varnothing$.

- There is a single sink place $s p$, where $s p \in S_{B}$, 
$s p^{\bullet}=\varnothing$.

- Every node $n$ in the instance net is on the path from $i p$ to $s p$.

1) $Z_{\text {cancel }} \subseteq Z_{B}$ : is a set of zero places $\left\{z_{\text {cancl }}, \ldots, z_{\text {cancx }}\right\}$, such that, $\forall z_{\text {canci } i} \in Z_{\text {cancel }}, z_{\text {canc }}{ }^{\circ}=\left\{\right.$ canc $\left._{i}\right\}$

2) $S_{I A R} \subseteq P$. $S_{I A R}$ is a set of places. $S_{I A R}=\left\{p \mid\left(p \in S_{B}\right)\right.$ $\wedge\left(p=n \mid n \in\left\{A D_{I A R} \cap\left\{B N \cup O N-\left\{p_{o i} \mid \exists o f \in O F\right.\right.\right.\right.$ and of $=o_{\mathrm{i}} \times o_{i^{\prime}}$ and $\neg \exists t b$ on of $\} \cup\{i N, f N\} \cup\left\{p_{c} \mid c \in\right.$ $C F\}\}\}$

3) Cancel $\subset T$ : a set of special transitions $\left\{\right.$ canc $_{1}, \ldots$, $\left.c a n c_{x}\right\}$, such that, the enabling condition to each transition canc $_{\mathrm{i}}$ is the marking of $z_{\text {canci }}$ and the effective firing condition is the instantaneous marking calculated, when $z_{\text {canci }}$ is marked. Given a transition $\operatorname{canc}_{i}$, the firing sequence is given by:

$\left\{z_{\text {canci }}, \mathrm{M}_{\mathrm{SIAR}}\right\}\left(\right.$ canc $_{i}>\mathrm{M}^{\prime} / z_{\text {canci } i} \notin \mathrm{M}^{\prime} \wedge \mathrm{M}_{\mathrm{SIAR}}=\varnothing$ and - canc $_{\mathrm{i}}=\left\{\mathrm{S}_{\mathrm{IAR}}, z_{\text {canci }}\right\}$ where $\mathrm{M}_{\mathrm{SIAR}}$ and $\mathrm{M}_{\mathrm{SIAR}}$ respectively stand for $\mathrm{S}_{\mathrm{IAR}}$ markings before and after firing canc $_{i}$.

4) $Z S N$ : denotes a zero-safe net, i.e., $Z S N=\left(S_{B}, T_{B} ; F_{B}\right.$, $\left.W_{B}, u_{B} ; Z_{B}\right)$ as defined in Section 3.2 such that:

$S_{B}=B N \cup O N-\left\{p_{o \mathrm{i}} \mid \exists\right.$ of $=\left(p_{o i}, p_{o i+1}\right)$ and $\neg \exists$ tb on of $\} \cup\{i N, f N\} \cup\left\{p_{c} \mid c \in C F\right\}$

For each branch or object node, we create a place. When two object nodes are connected via an edge not carrying a transformation behavior, just one place is created and takes the name of one of the two (since they have the same name).

$T_{B}=E N \cup C N \cup\left\{t_{o \mathrm{i}} \mid \exists o f \in O F\right.$ and $o f=o_{\mathrm{i}} \times o_{i^{\prime}}$ and $\exists t b$ on of $\cup\left\{\left\{t_{d i d i^{\prime}} \mid \exists o f \in O F\right.\right.$ and $o f=d_{\mathrm{i}} \times d_{i^{\prime}}$ or $\exists c f \in$ $C F$ and $\left.c f=d_{\mathrm{i}} \times d_{i^{\prime}}\right\} \cup\left\{t_{m \text { im } i^{\prime}} \mid \exists o f \in O F\right.$ and $o f=m_{\mathrm{i}} \times m_{i^{\prime}}$ or $\exists c f \in C F$ and $\left.c f=m_{\mathrm{i}} \times m_{i^{\prime}}\right\} \cup$ Cancel.

Executable and control nodes are mapped into transitions. An object flow gives rise to a new transition iff this edge carries a transformation behavior. For each control flow, we define a transition.

$F_{B}=\left\{\langle\mathrm{x}, \mathrm{y}\rangle \mid\langle\mathrm{x}, \mathrm{y}\rangle \in C F \wedge\left(\mathrm{x} \in T_{B}\right) \wedge\left(\mathrm{y} \in S_{B}\right)\right\} \cup$ $\left\{\langle\mathrm{x}, \mathrm{y}\rangle \mid\langle\mathrm{x}, \mathrm{y}\rangle \in C F \wedge\left(\mathrm{x} \in S_{B}\right) \wedge\left(\mathrm{y} \in T_{B}\right)\right\} \cup\{\langle\mathrm{x}, \mathrm{y}\rangle \mid(\mathrm{x}$ $\left.\left.\in T_{B}\right) \wedge\left(\mathrm{y} \in S_{B}\right) \wedge \exists A_{i} \mid \mathrm{x}=A_{i} \wedge \mathrm{y}=o_{i^{\prime}}\right\}$.

$W_{B}: F_{B} \rightarrow 1 \mathrm{~N}$.

$i p=i N$.

$s p=f N$.

$u_{B}=\{i N\}$

$Z_{B}=\{e v t\}, z_{\text {canc }}=e v t$.

The above definition, mapping an $\mathrm{AD}_{\mathrm{IAR}}$ to a $\mathrm{ZSN}$, is faithful to the intuitive mapping given in Table 2. Concurrent regions, streams, and exceptions are not yet taken into account. The semantics of cancellation is deeply considered. So far, none of the previous works authors has considered the problem of reactivity in ADs cancellation behavior.

\section{Conclusions}

This paper is a continuation of our last two papers [16],
[17]. Their main goal was to propose a generic mapping of ADs basic concepts to ZSNs ones. Especially, they handle formalization of concurrent-region, while considering the traverse-to-completion semantics and exception outputs streaming parameters via ZSNs.

This paper highlights also the failure of Petri nets to cover high semantics of ADs, namely the Interruptible Activity Region. Here, we have proposed, with the same spirit, the use of ZSNs as a formal semantic framework to handle this region.

A generic mapping from ADs to ZSNs, covering basic constructs, concurrent-region, traverse-to-completion principle, streaming parameters, exception outputs and the Interruptible Activity Region has been defined. Its formal definition based on ZSNs covers until now control flow, data flow and Interruptible Activity Region. Concurrent region, streaming parameters and exceptions are not yet covered, but they can be integrated very simply in the defined $Z S N_{I A R}$.

Some other constructs namely expansion-region and exception handling are to be considered in future works. Our aim is to define an EZS-Net (Extended Zero-Safe Net) for all new constructs defined in AD2. The EZS-Net will be dedicated to formalize UML ADs in a complete and unified way.

ZSNs are tile logic based models which is an extension of rewriting logic, taking into account the concept of side effects and dynamic constraints on terms. Mapping UML2 ADs to ZSNs can be followed by the projection of these latter in rewriting logic and thus, exploiting its practical system Maude for verification and validation aims.

\section{REFERENCES}

[1] “OMG Unified Modelling Language: Superstructure," Final Adopted Specification Version 2.0,Technical Report, Object Management Group, November 2003. http://www .omg.org

[2] T. Schttkowsky and A. Föster, "On the Pitfalls of UML 2 Activity Modeling," International Workshop on Modeling in Software Engineering, Minneapolis, IEEE Computer Society, 2007.

[3] H. Störrle and J. H. Hausmann, "Towards a Formal Semantics of UML 2.0 Activities," Software Engineering Vol. 64, 2005, pp. 117-128.

[4] T. Murata, "Petri Nets: Properties, Analysis and Applications," Proceedings of the IEEE, Vol. 77, No. 4, April 1989, pp. 541-580.

[5] E. Borger and R. Stark, "Abstract State Machines," Springer Verlag, 2003.

[6] R. Bruni and U. Montanari, "Zero-Safe Nets, or Transition Synchronization Made Simple," In C. Palamidessi and J. Parrow, Eds., Proceedings of the 4th workshop on Expressiveness in Concurrency, Electronic Notes in Theoretical Computer Science, Santa Margherita 
Ligure, Elsevier Science, Vol. 7, 1997.

[7] J. P. Barros and L. Gomes, "Actions as Activities and Activities as Petri Nets," In Jan J"urjens, Bernhard Rumpe, Robert France, and Eduardo B. Fernandey, Eds., UML 2003 Workshop on Critical Systems Development with $U M L$, San Francisco, 2003, pp. 129-135.

[8] T. S. Staines, "Intuitive Mapping of UML 2 Activity Diagrams into Fundamental Modeling," "Concept Petri Net Diagrams and Colored Petri Nets," 15th Annual IEEE International Conference and Workshop on the Engineering of Computer Based Systems, Belfast, 2008.

[9] H. Störrle, "Semantics of Exceptions in UML 2.0 Activities," Journal of Software and Systems Modeling, 9 May 2004. www.pst.informatik.uni-muenchen.de/stoerrle

[10] H. Störrle, "Semantics of Control-Flow in UML 2.0 Activities," In N.N. Ed., Proceedings IEEE Symposium on Visual Languages and Human-Centric Computing, Rome, Springer Verlag, 2004.

[11] H. Störrle, "Semantics and Verification of Data Flow in UML 2.0 Activities," Electronic Notes in Theoretical Computer Science, Vol. 127, No. 4, 2005, pp. 35-52. www. pst.informatik.uni-muenchen.de/-stoerrle

[12] H. Störrle, "Semantics and Verification of Data-Flow in UML 2.0 Activities," Proceedings International Workshop on Visual Languages and Formal Methods, IEEE Press, 2004, pp. 38-52. www.pst.informatik.uni-muench en.de/_stoerrle

[13] R. Eshuis and R. Wieringa. "Comparing Petri Net and Activity Diagram Variants for Workflow Modelling-A Quest for Reactive Petri Nets," In Weber et al. Petri Net Technology for Communication Based Systems, Lecture
Notes in Computer Science, Vol. 2472, 2002, pp. 321-351.

[14] R. Eshuis and R. Wieringa. "A Real-Time Execution Semantics for UML Activity Diagrams," In H. Hussmann, Ed., Fundamental Approaches to Software Engineering, Lecture Notes in Computer Science, Genova, Springer Verlag, Vol. 2029, 2001, pp. 76-90.

[15] R. Eshuis and R. Wieringa. "An Execution Algorithm for UML Activity Graphs," Proceedings of the 4th International Conference on The Unified Modeling Language, Modeling Languages, Concepts, and Tools, Lecture Notes in Computer Science, Toronto, Springer Verlag, Vol. 2185, 2001, pp. 47-61.

[16] S. Boufenara, F. Belala and C. Bouanaka, "Les Zero-Safe Nets Pour la Préservation de la TTC Dans les Diagrammes d'activité d'UML," "Revue des Nouvelles Technologies de l'Information RNTI-L-3," Cépaduès éditions, $15^{\text {ème }}$ Conférence Internationnale sur les Langages et Modèles à Objets : LMO, 2009, pp. 91-106.

[17] S. Boufenara, F. Belala and N. Debnath, "On Formalizing UML 2.0 Activities: Stream and Exception Parameters," 22nd International Conference on Computers and Their Applications in Industry and Engineering CAINE-2009, San Francisco, 4-6 November 2009.

[18] C. Bock, "UML 2 Activity and Action Models," Part 6: Structured Activities, 2005. http://www.jot.fm/issues/issue _2005_05/column4

[19] R. Bruni and U. Montanari, "Transactions and Zero-Safe Nets,” In: H. Ehrig, G. Juhás, J. Padberg and G. Rozenberg, Eds., Proceedings of Advances in Petri Nets: Unifying Petri Nets, Lecture Notes in Com- puter Science, Springer Verlag, Vol. 2128, 2001, pp. 380-426. 